\title{
Body position alters human resting-state: Insights from multi-postural magnetoencephalography
}

\author{
Robert T. Thibault, Michael Lifshitz, \& Amir Raz \\ Brain Imaging and Behavior. 2015 Sep 26 [epub] \\ doi: \\ $10.1007 / \mathrm{s} 11682-015-9447-8$ \\ Copyright 2015
}




\title{
Body position alters human resting-state:
}

\section{Insights from multi-postural}

\section{magnetoencephalography}

\author{
Robert T. Thibault ${ }^{\mathrm{a}}$, Michael Lifshitz ${ }^{\mathrm{a},}$ Amir Raz ${ }^{\mathrm{a}, \mathrm{b} *}$ \\ ${ }^{a}$ McGill University, 3775 University Street, Montreal, QC, H3A 2B4, Canada. \\ ${ }^{\mathrm{b}}$ The Lady Davis Institute for Medical Research \& Institute for Family and Community \\ Psychiatry, Jewish General Hospital, 4333 Cote Ste. Catherine Road, Montreal, QC, H3T 1E4, \\ Canada.
}

*Please address correspondence to:

Professor Amir Raz, 4333 Cote Ste. Catherine Road, Montreal, QC, H3T 1E4, Canada.

Email: amir.raz@mcgill.ca

Tel: $1-514-340-8210$

Fax: 1-514-340-8124 


\begin{abstract}
Neuroimaging researchers tacitly assume that body-position scantily affects neural activity. However, whereas participants in most psychological experiments sit upright, many modern neuroimaging techniques (e.g., fMRI) require participants to lie supine. Sparse findings from electroencephalography and positron emission tomography suggest that body position influences cognitive processes and neural activity. Here we leverage multi-postural magnetoencephalography (MEG) to further unravel how physical stance alters baseline brain activity. We present resting-state MEG data from 12 healthy participants in three orthostatic conditions (i.e., lying supine, reclined at $45^{\circ}$, and sitting upright). Our findings demonstrate that upright, compared to reclined or supine, posture increases left-hemisphere high-frequency oscillatory activity over common speech areas. This proof-of-concept experiment establishes the feasibility of using MEG to examine the influence of posture on brain dynamics. We highlight the advantages and methodological challenges inherent to this approach and lay the foundation for future studies to further investigate this important, albeit little-acknowledged, procedural caveat.
\end{abstract}

Keywords: MEG, neuroimaging, posture, supine position, upright position 


\section{BACKGROUND}

Cognitive neuroscientists rarely consider the influence body position wields on brain activity; and yet postural discrepancies hold important implications for the acquisition and interpretation of neuroimaging data (Raz et al., 2005). Moreover, converging evidence demonstrates that posture regulates physiological factors, including hemodynamics, and influences concomitant neurocognitive processing (Cole 1989; Lipnicki and Byrne 2005; Lundström et al. 2008; Ouchi et al. 2001; Spironelli and Angrilli 2011). Such orthostatic variables take on particular significance as the field moves toward triangulating resting-state data from multiple imaging modalities involving different body stances (Agam et al. 2011). For example, whereas most functional magnetic resonance imaging (fMRI) scanners require participants to lie supine, occipital sensors impede pristine supine recordings with electroencephalography (EEG). Here we propose MEG as a promising imaging modality for elucidating how posture influences the temporal and spatial dynamics of the living human brain.

While a handful of fMRI studies report how environmental and contextual variables such as eye closure and gaze fixation alter the activity of resting state networks (RSNs) - i.e., networks of distributed brain regions demonstrating coherent activity at rest (Deco et al. 2011; Yan et al. 2009) — these accounts shy away from addressing body-position as a potential caveat.

Posture likely influences the functional architecture of the resting brain (Lipnicki and Byrne 2008; Lundström et al. 2008). Comparing postures using a stance-adjustable positron emission tomography (PET) gantry, studies have reported signal differences across a wide range of cortical and subcortical regions (Ouchi et al. 2001, 2005). In addition, a few studies have found changes in EEG as a function of posture (Chang et al. 2011; Rice et al. 2013). Recent EEG findings, moreover, indicate that changes in orthostatic condition rapidly influence high- 
frequency electrical activity across the cortex (Thibault et al. 2014). In terms of physiology, gravity in the supine position stimulates baroreceptors that reduce sympathetic system activation (Mohrman and Heller 2003), decreasing noradrenergic output from the locus coeruleus (Berridge and Waterhouse 2003) and consequently dampening cortical excitability (Rau and Elbert 2001). Furthermore, regardless of age, the supine posture associated with fMRI modulates respiration by altering diaphragm function (Rehder 1998). This caveat holds special importance for confounds associated with independent component analysis (ICA)-based RSN measures (Birn et al. 2008). Such postural nuances come to the fore as researchers begin to compare supine fMRI findings with resting-state electrophysiological data from EEG and intracranial recordings typically acquired in the upright position (Agam et al. 2011; Lei et al. 2011, 2012). Thus, understanding how posture alters resting-state brain activity permits a more judicious way to reconcile findings from disparate neuroimaging modalities and binds procedural nuances to the scientific investigation of neural processes.

MEG scanners permit recording while sitting upright, reclining at a $0-45^{\circ}$ angle, or lying supine - an advantageous feature for characterizing neural patterns associated with body position. In contrast, although upright MRI scanners for humans exist, they tend to employ lower magnetic fields, which often preclude functional sequences. Furthermore, while previous posture studies employed either adjustable-gantry positron emission tomography (PET) or EEG, these methodologies lack integration of spatial and temporal signals. Whereas PET provides good (millimetric) spatial resolution but low temporal resolution, EEG offers millisecond temporal resolution but poor signal localization due to smearing of electrical signals when traveling through cephalic tissues to the scalp. In an EEG context, the highly conductive cerebral spinal fluid (CSF) causes a shunting effect that dampens the magnitude of electrical signals recorded at 
the scalp (Ramon et al. 2004; Rice et al. 2013; Wendel et al. 2008). In addition, this shunting effect propagates electrical currents through the CSF tangential to the scalp (Wolters et al. 2006). Because this tangential electrical current runs perpendicular to EEG electrodes, it exerts a negligible effect on the EEG signal. This electrical current, however, produces a circular magnetic fields that reaches MEG sensors. Thus, while magnetometers and gradiometers measure the intracellular currents from the dendrites of pyramidal cells both parallel and tangential to the scalp (Hillebrand and Barnes 2002; Okada et al. 1997), they also record small additional magnetic fields originating from shunted currents in the CSF (Vorwerk et al. 2014). Otherwise, CSF and cephalic tissues leave magnetic signals emitted from the brain relatively undisturbed. Compared to EEG, therefore, in a MEG context CSF exerts an opposite effect on the magnitude of recorded brain signals (Vorwerk et al. 2014) - slightly increasing, rather than largely decreasing, the signal amplitude. Thus, MEG provides a useful complement to EEG studies of posture. In addition, advances in MEG source-localization and connectivity analysis permit fine-grained examination of temporospatial dynamics in the resting brain (de Pasquale et al. 2010). Such novel analytic approaches reveal MEG connectivity networks spatially congruent with classical fMRI RSNs. Furthermore, MEG may eventually permit researchers to examine temporal nuances otherwise difficult to probe with fMRI, including non-stationary dynamics among and within intrinsic connectivity networks — an emerging topic in resting-state research (de Pasquale et al. 2010; Jones et al. 2012). Comparing body positions with MEG, therefore, presents a powerful means of elucidating postural determinants of resting brain activity.

Here we present pilot findings from a MEG study comparing resting-state activity in three body postures — sitting upright, reclining at $45^{\circ}$, and lying supine. We hypothesized that our MEG findings would mirror previous multi-postural EEG results in which participants 
demonstrated widespread increases in beta and gamma activity in more upright postures (Chang et al. 2011; Cole 1989; Thibault et al. 2014). We highlight methodological issues inherent to this approach and explain how to control for such potential caveats. We submit our sensor-level analysis as proof-of-concept to encourage future analytic efforts to further unravel the influence body position imparts to resting-state network activity.

\section{MATERIALS AND METHODS}

\section{Participants}

Twelve participants (mean age $=26.4 \pm 4.2$ years; six females) provided written informed consent in accordance with the Research Ethics Board at the Montreal Neurological Institute and in compliance with the Code of Ethics of the World Medical Association - Declaration of Helsinki. Participants were right-handed, reported normal or corrected-to-normal vision, and received monetary compensation for their involvement.

\section{Procedure}

All sessions began with a 2-minute empty-room MEG recording. We then tested participants for magnetic artefacts in a brief preliminary MEG scan. Participants transitioned among three postures (sitting upright, sitting reclined at $45^{\circ}$, and lying supine; see Fig. 1) in a counterbalanced fashion. For each posture, participants underwent two eight-minute resting-state MEG scans separated by a brief (1-2 minute) verbal-response questionnaire concerning subjective experiences in the scanner (the present paper does not address the questionnaire data). Before each run we instructed participants to relax, stay still, and fixate on a point directly ahead while keeping their eyes open. We employed the eyes-open, rather than eyes-closed, condition to best match the present imaging context to everyday waking environments. Notably, recent 
findings from resting-state fMRI and EEG experiments demonstrate that the human brain assumes different default states when eyes are open rather than closed (Thibault et al. 2014; Xu et al. 2014). Accordingly, an eyes-closed paradigm may produce distinct results from the present eyes-open experiment. We standardized the visual environment by draping a white sheet around the immediate visual field.

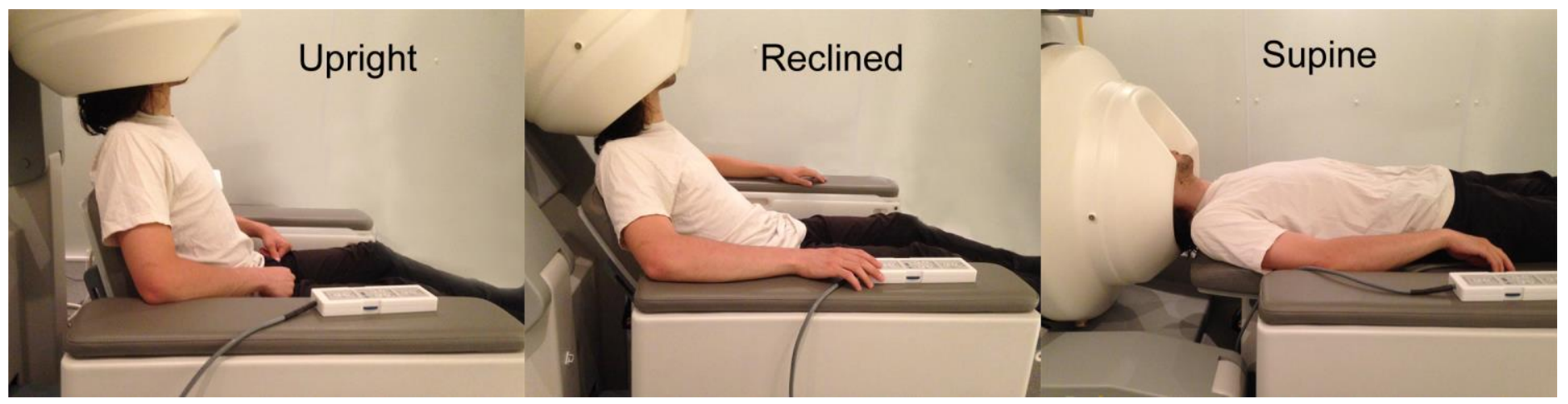

Fig. 1 Posture and dewar positions

\section{Magnetoencephalography}

We used the VSM/CTF system (MEG International Services Ltd.) at the Montreal Neurological Institute (McGill University, Quebec, Canada). The sensor array consisted of 275 axial gradiometers and an additional nine reference magnetometers and 17 reference gradiometers farther from the helmet to remove environmental noise. Recording used a sampling rate of $2400 \mathrm{~Hz}$ inside a magnetically shielded room (i.e., full 3-layer passive shielding). We used head-positioning coils and a 3-D digitizer system (Polhemus Isotrack) to register head position throughout. In line with standard guidelines, we recorded electrocardiograms (ECG) and electrooculograms (EOG) to capture heartbeat and eye-blink artifacts (Gross et al. 2013). Between postures participants left the scanning room while an experimenter adjusted the angle of the MEG dewar. We then waited 15 minutes to ensure that the liquid helium level outside the helmet had equalized and proceeded to conduct a two-minute empty-room recording to detect 
environmental noise. Based on tests conducted on the MEG system we used at the Montreal Neurological Institute, noise contamination from the sensors levels off within 15 minutes. While the helium boil-off rate increases when the dewar is horizontal, all sensors remain submerged in liquid helium and the temperature at each sensor is constant.

\section{Data Processing}

We processed and analyzed MEG data using Brainstorm (Tadel et al. 2011). Following the manufacturer's standard pre-processing (third-order gradient compensation), we applied a high-pass filter at $0.1 \mathrm{~Hz}$ and removed potential electrical contamination using a sinusoidal (notch) filter at 60, 120, 180, and $240 \mathrm{~Hz}$. We then used the eye movement detection processes from Brainstorm to mark blink events based on EOG recordings from each participant. We designed a standard signal-space projector (SSP) in the 1.5-15 Hz frequency range and within $\pm 200 \mathrm{~ms}$ of blink events to remove contamination from eye artifacts. Next, we discarded all data segments in which either of the two head localizer coils (left and right pre-auricular points) was farther than $5 \mathrm{~mm}$ from its position at the beginning of the recording. We set the threshold for excessive head motion at $5 \mathrm{~mm}$. We chose this value in line with previous research (Brookes et al. 2011; Johnson et al. 2010; Moradi et al. 2003; Poghosyan and Ioannides 2007; Xiang et al. 2014) and the spatial precision of MEG, which lies around $5 \mathrm{~mm}$ (Moradi et al. 2003). We then detected heartbeats with an ECG recording and applied a standard SSP (13-40 Hz, $\pm 40 \mathrm{~ms})$ based on heartbeats events that occurred at least $250 \mathrm{~ms}$ from blink events to remove cardiac artifacts. Next, we visually inspected all data for muscle artifacts and discarded segments with transient high-amplitude and broadly distributed high-frequency activity. Lower-amplitude, sustained muscle activity persists in some recordings as is common in EEG and MEG data (Muthukumaraswamy 2013). Lastly, we employed Brainstorm to calculate the average power- 
spectrum density (PSD) on each of the 275 MEG sensors, for delta $(\delta) 2-4 \mathrm{~Hz}$, theta $(\theta) 4-8 \mathrm{~Hz}$, alpha $(\alpha)$ 8-14 Hz, beta ( $\beta$ ) 14-30 Hz, low-gamma $(\gamma 1) 30-58$, and high-gamma $(\gamma 2) 62-90 \mathrm{~Hz}$ using $50 \%$ overlapping windows of two-seconds epochs. Here we conducted a sensor level analysis to extend our previous EEG effort (Thibault et al. 2014) and provide a direct comparison using MEG.

\section{Statistical Analysis}

Using Statistical Analysis Software 9.3 (SAS $®$ ), we performed two repeated measures full-factorial analyses of variance (ANOVA) on the logarithm of the absolute power (measured in $\mathrm{fT}^{2}$ ) at each sensor for each bandwidth: (1) a two-way ANOVA (Posture $\mathrm{x}$ Run) on the data collected from participants, and (2) a one-way ANOVA (Posture) for the empty-room recordings. To account for multiple comparisons, we calculated an adjusted p-value (q-value) for each dimension of the ANOVA at each bandwidth using positive false discovery rate (Storey, 2002). We corrected all pairwise comparisons using Tukey's Honest Significant Difference Test (Westfall and Tobias 1999). Using SAS ${ }^{\circledR}$ we confirmed normality and homogeneity of variance in each analysis.

\section{RESULTS}

We found a main effect of posture on high-gamma activity over left frontal and left temporal cortex (Fig. 2). High-gamma power increased in these regions when sitting compared to when reclined or supine (Fig. 2 C-D), but did not differ between reclined and supine postures (Fig. 2 E). Differences in delta, theta, alpha, beta, and low-gamma activity between postures lacked significance. We found no difference between the two runs in each posture for any bandwidth. 


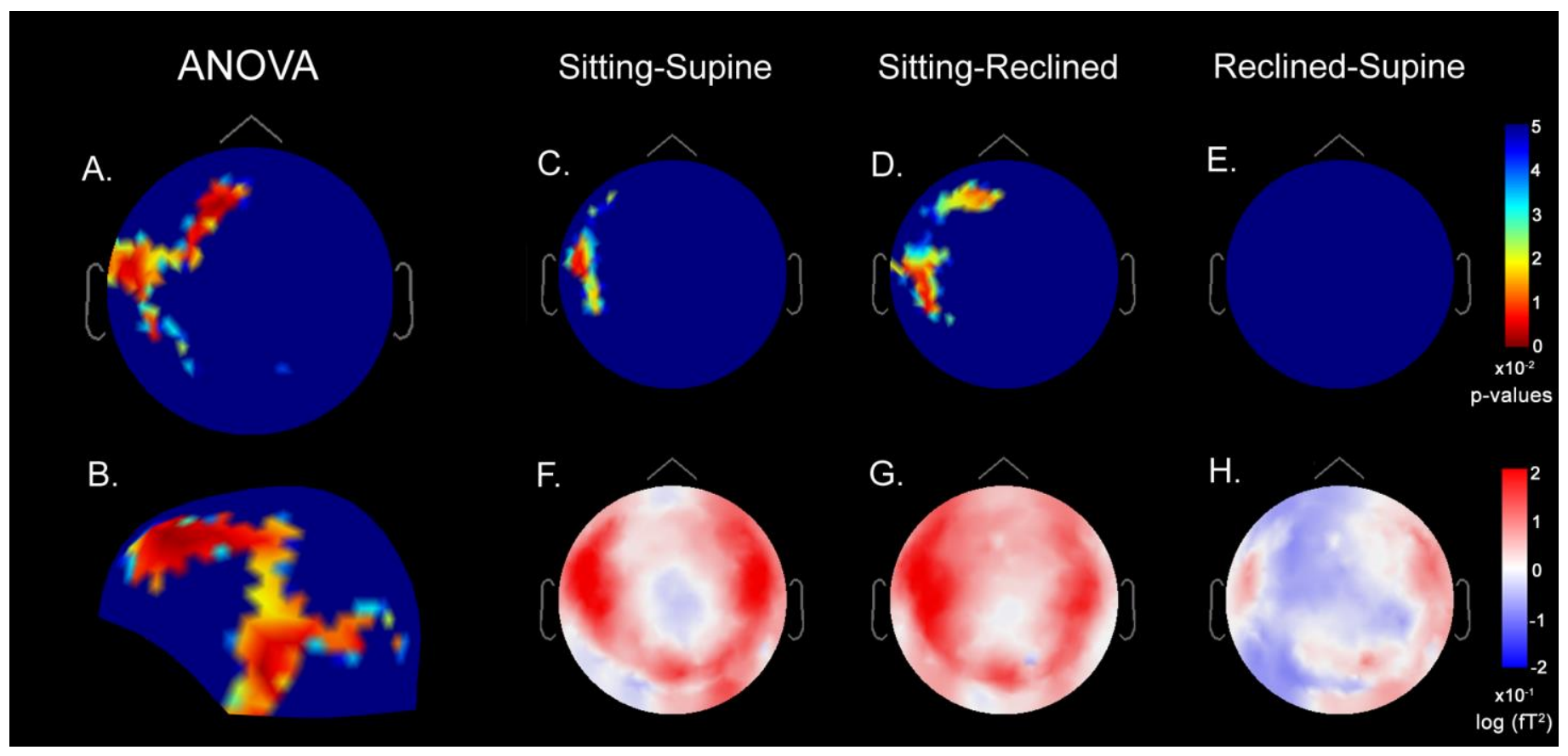

\section{Fig. 2 High-gamma activity differs across postures}

A. Color map depicting scalp regions where two-way ANOVAs yielded statistically significant (red: $\mathrm{p}<.01$ to light blue: $\mathrm{p}<.05$ ) and non-significant changes (dark blue: $\mathrm{p}>.05$ ) across postures. B. The same ANOVA results mapped onto a 3-dimensional depiction of the MEG helmet (viewed from the left side). C-E. Heat maps shows Tukey-corrected pairwise comparisons between specific postures. Any color other than dark-blue represents an increase in power while sitting upright. F-H. Maps depicts the average power differences between specific postures. Red indicates an increase in power when more upright whereas blue indicates a decrease in power when more upright.

In the empty-room recordings, ANOVAs revealed a main effect of dewar position on seven of the 275 sensors in the high-gamma range only (Fig. 3). Two of the sensors displaying significant changes between postures in the empty-room recordings also showed significance in the participant analysis. However, pairwise Tukey-comparisons revealed that in the case of 
empty-room recordings, statistically significant differences arose between supine and reclined postures only, whereas in the participant analysis changes occurred between sitting and supine or sitting and reclined, but not between supine and reclined.

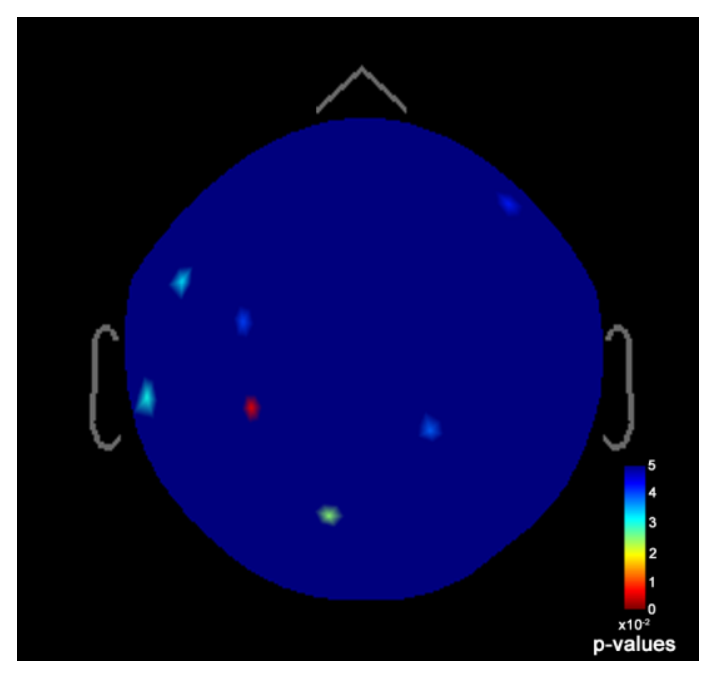

\section{Fig. 3 Empty-room ANOVA results}

This figure shows the seven sensors that differed in high-gamma power across dewar positions in the empty-room recordings.

\section{DISCUSSION}

We present the first demonstration of using MEG to compare resting-state brain activity in multiple postures. Our results suggest that variations in posture perturb resting-state neurophysiology. High-gamma $(62-90 \mathrm{~Hz})$ activity increased over left frontal and left temporal regions when participants sat upright compared to when sitting reclined or lying horizontal (Fig. 2). The first and second runs were comparable, suggesting a change in baseline activity rather than a transient event-mediated effect. These findings have direct relevance for comparisons between upright EEG recordings and supine fMRI scans. EEG experiments have implicated gamma band activity in a host of cognitive processes including attention and memory (Jensen et 
al. 2007). The BOLD signal, which serves as a proxy for neural activity, as ascertained by fMRI measurements, correlates tightly with synchronized gamma activity (Niessing et al. 2005; Nir et al. 2007; Shmuel and Leopold 2008). Our demonstration that postural manipulation is sufficient to amplify spontaneous neural activity warrants caution in interpreting results between imaging modalities that tend to employ different postures (i.e., EEG and fMRI). In particular, these results likely bear on studies examining resting state recordings of neural activity. Researchers might uncover distinct RSNs based on the posture assumed in a given experiment. To overcome this potential caveat, researchers attempting to compare EEG, MEG, and fMRI data could conduct simultaneous recordings with an MRI compatible EEG system or record MEG in the supine posture. Complementing previous accounts from EEG (Chang et al. 2011; Thibault et al. 2014) and PET (Ouchi et al. 2001, 2005), the present findings indicate that resting-state neuroimaging data differs when sitting upright compared to when lying down supine.

Of the various physiological mechanisms contributing to high-gamma activity, our data suggest that changes in local cortical activity may account for the posture-mediated differences recorded at the sensors. In addition, contamination due to muscle activity might also contribute to our recordings because it pervades EEG and MEG signals in the gamma range (Muthukumaraswamy 2013). Frontalis muscles peak around 20-30 Hz and temporalis muscles at 40-80 Hz (Goncharova et al. 2003). Based on the unilaterality of our results, however, it appears unlikely that muscle contamination accounts for the present results. Examining the differences in high-gamma activity between postures at the individual level reveals that bilateral frontal, temporal, and occipital activity typical of muscles artifacts (Goncharova et al. 2003) is largely absent (Fig. 4). Nonetheless, unilateral significance does not imply significance of unilaterality. Thus, we cannot completely rule out muscle contamination as a contributing factor. Indeed, 
visual inspection of Figure 2 (F-G) intimates that high-gamma increased in both hemispheres when more upright; yet, this effect was smaller and statistically non-significant in the right hemisphere. We recently ran a comparable experiment using EEG and EMGs and found that posture influenced neither lateral neck and superior jaw muscles (sternocleidomastoid and masseter) nor muscles superior, lateral, and inferior to the eye (frontalis and orbicularis oculi) (Thibault et al. 2014). While in that previous study an EMG placed on the trapezius recorded an increase in muscle activity when sitting upright, we would not expect frontal and temporal artifacts to originate from the trapezius. Instead we would expect such potential muscle contamination to generate a gradient of postural EEG effects - greatest around occipital regions and diminishing further away, weakest towards frontal areas. However, our data are inconsistent with this pattern (Fig. 2).

One recent EEG study (Rice et al. 2013) postulated that posture-mediated changes in CSF thickness may be the key mechanism underlying alterations in gamma oscillations. In the present experiment, however, we observed significant MEG changes over the left hemisphere only; yet, body-tilt is unlikely to prompt unilateral changes in CSF thickness across participants. Indeed, heat maps comparing postures within individual participants lack a clear bilateral effect we might have expected if differences in CSF influenced the MEG signal (Fig. 4). As we discuss above, nonetheless, unilateral significance does not necessarily imply significance of unilaterality. 


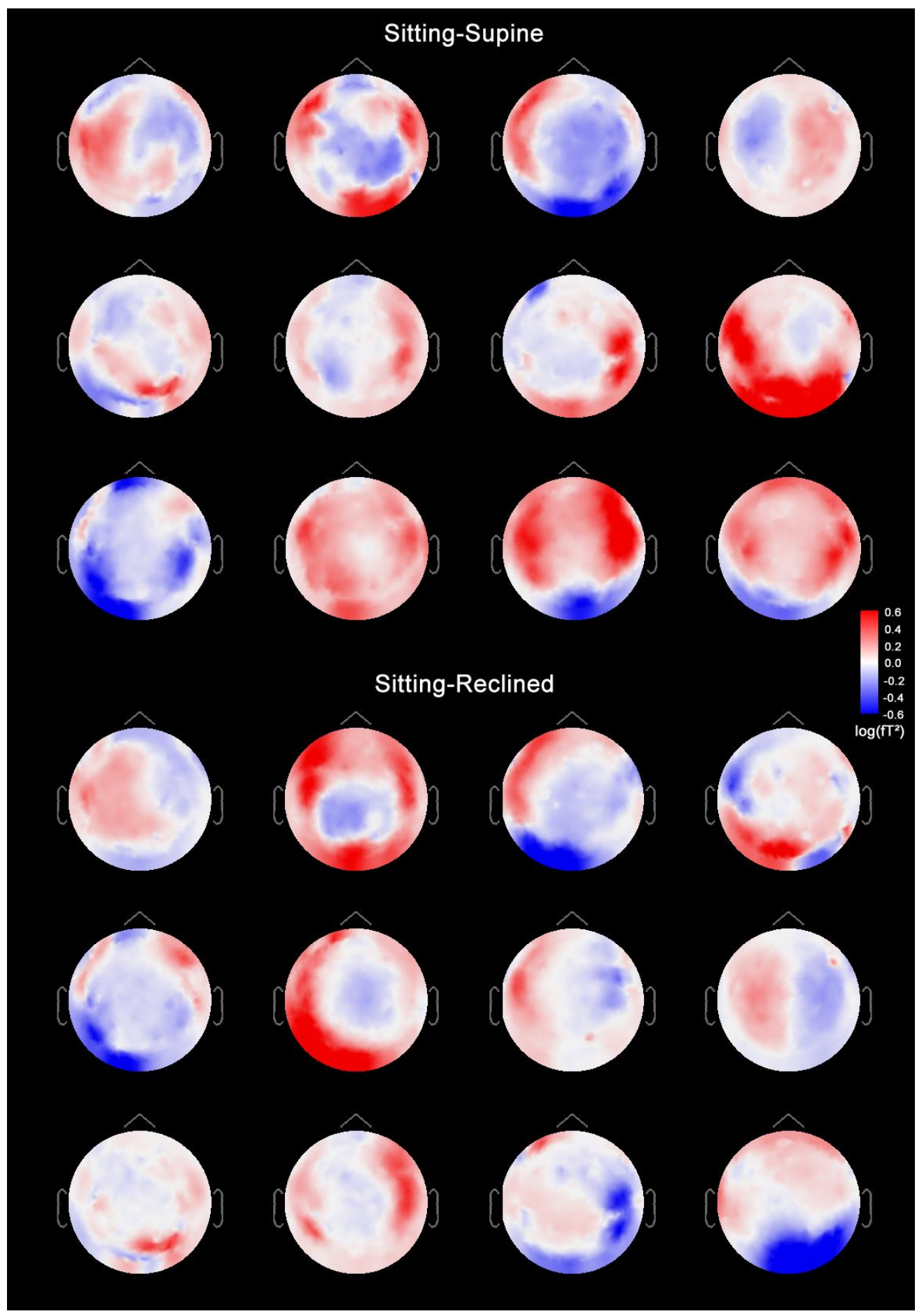

Fig. 4 Individual differences in high-gamma

Heat maps depict the difference in high-gamma power for each of the 12 participants between sitting and supine postures and sitting and reclined postures. 
The relationship between CSF thickness and multi-posture MEG is poorly understood. While CSF thickness distorts the topography of electric and magnetic signals comparably, this cephalic fluid increases the magnitude of MEG data only slightly while decreasing the magnitude of EEG data substantially (Vorwerk et al. 2014). One experiment leveraged upright and recumbent MRI scanners to demonstrate that total intracranial CSF decreases when upright compared to when supine as gravity draws fluids downward into the spinal canal (Alperin et al. 2005). Given these previous findings, we might expect a widespread decrease in MEG gamma activity when upright, paralleling the decrease in total intracranial CSF. However, our results diverge from this pattern (Fig 2). Another recent study highlighted the spatial specificity of posture-related CSF thinning. This account demonstrated that, when supine, occipital CSF thins by up to $30 \%$ compared to when prone (Rice et al. 2013). However, this study did not include an upright condition, likely due to the sparse availability of erect MRI scanners. Thus, the relative thickness of frontal and occipital CSF between upright and supine postures remains elusive. Multi-posture simultaneous EEG/MEG recordings could shed light on how body position and CSF shifting differently affect magnetic and electric brain signals.

We observed postural effects over the inferior and dorsolateral frontal gyri, the lateral sulcus, and the supramarginal gyrus of the left hemisphere across participants (Fig. 2B) and within almost all individuals (Fig. 4). These cortical regions contain major speech centers, including Broca's and Wernicke's areas, as well as auditory cortex. As all our participants were right-handed, lateralized results may suggest that posture influenced language faculties. Yet, the precise locus of activity change remains undetermined as sensors lateral to, rather than above, the neuronal source measure the field maxima (Bastiaansen and Knösche 2000). 
Future experiments incorporating source-level analysis, phenomenological experiencesampling, or behavioral tasks, will likely further unravel the intricacies underlying multi-postural brain data. We hope to report on such efforts before long. Taken together, our multi-posture MEG findings corroborate previous EEG and PET reports and highlight posture as a determinant of neuroimaging data.

\section{Caveats}

The present MEG results differ from previous EEG data collected from similar experiments. While EEG efforts demonstrated high-gamma, low-gamma, and beta modulation, our MEG analysis revealed high-gamma alterations only. Whereas EEG electrodes rest directly on the scalp and thus limit variations in brain-to-electrode distance across participants, MEG brain-to-sensor distances range widely depending on head size and position in the helmet. As the magnetic field decays exponentially with distance from the source, brain-to-sensor distance may encourage large inter-subject and inter-run variance, potentially masking posture-mediated power modulation. Thus, the present MEG results may depict only the most salient effects observed in previous multi-postural EEG experiments. We attempted to control for this variable by co-registering head placement relative to helmet position for all 72 runs (12 participants x 3 postures $\mathrm{x} 2$ runs). We found that current software, however, is unable to effectively transform data with notable variations in brain-to-sensor distance across 72 runs. Nonetheless, differences in brain-to-sensor distance across postures are unlikely to account for our findings because we observed largely unilateral changes. Decreased brain-to-sensor distance for the left hemisphere would correspond to increased brain-to-sensor distance for the right hemisphere. Under such circumstances we would expect opposing directions of high-gamma modulation between hemispheres, a pattern incongruent with our group level results (Fig. 2 F-G). At the individual 
level, however, a few heat maps show opposing differences in high-frequency power between postures in the right and left hemispheres (Fig. 4). A slightly lateralized head position between postures may have contributed to these participant heat maps. Between-subject variations in brain-to-sensor distance likely decreased statistical power and may have masked potential difference in magnetic activity. In the future, more advanced helmet co-registration algorithms would likely permit explorations of sensor-level effects. Source analyses, which register data from multiple runs to a common source-space referential, would also serve to reveal additional spatial information.

Head motion might also affect our results. To account for this possibility, we excluded epochs with head positions greater than $5 \mathrm{~mm}$ from the initial position. Notably, excessive head displacement occurred in only three sitting runs and one reclined run. Some participants may slouch during recording in upright and reclined positions, thus causing a substantial shift in head position (Gross et al. 2013). This finding suggests the supine posture may best serve researchers aiming to minimize head movements. Moreover, whereas in certain neuroimaging contexts small head movements, even those that survive standard motion correction, can generate spurious resting-state findings (e.g., fMRI functional connectivity; Power et al. 2012), in the present MEG context head movements likely increased variance in the topography and amplitude of the MEG signal and decreased statistical sensitivity (Stolk et al. 2013). Such minor head movements may have obfuscated the effects we previously reported in an EEG context wherein electrodes retain a consistent position directly against the scalp (Thibault et al. 2014).

The angle of the head compared to the MEG helmet may have differed slightly across postures. Variation in head orientation can alter the geometric relationship between a specific sensor and the underlying anatomy (i.e., the position of a specific sulcus relative to the sensor) 
and consequently impact the amplitude of the measured signals (Okamoto et al. 2004). In future efforts, standardizing head position across postures and across participants using head localizer coils may help obviate this potential caveat.

Finally, as all our participants were right handed, muscle contamination and head position may have introduced different artifacts at sensors above the right and left hemispheres. Yet, any effect of handedness on the laterality of muscle activity or head position would likely persist within a given participant across all postures. Such an effect would have had a negligible impact on our results because we analyzed MEG differences across postures, not between hemispheres. However, if right-handed people consistently increase unilateral muscle tension, or tilt their heads to one side, when lying down but not when sitting upright, this would present a potential confound. We are not familiar with any research supporting this possibility.

\section{CONCLUSIONS}

Our present effort demonstrates how MEG can illuminate the influence posture wields on the resting human brain. MEG affords a powerful means of comparing multiple body positions in the same imaging modality. Our piece addresses the methodological issues inherent to neuroimaging studies of posture and highlights the benefits of our approach. Furthermore, we present a sensor-level analysis, laying the foundation for follow-up analytic efforts to further probe how body position alters fine-grained oscillatory dynamics within the resting brain. Unlocking the influence of posture on neural processing would account for the orthostatic parameters associated with distinct scanning environments and pave the road to a more scientific understanding of this pervasive, albeit little acknowledged, procedural nuance. 


\section{ACKNOWLEDGEMENTS}

Dr. Amir Raz acknowledges funding from the Canada Research Chair program, Discovery and Discovery Acceleration Supplement grants from the Natural Sciences and Engineering Research Council of Canada (NSERC), Canadian Institutes of Health Research, and the BIAL Foundation. Robert T. Thibault acknowledges a Fonds de recherche du Québec - Nature et technologies (FRQNT) graduate scholarship and an Alexander Graham Bell Canada Graduate Scholarship from NSERC. Michael Lifshitz acknowledges a Francisco J. Varela Research Award from the Mind and Life Institute and a Vanier Canada Graduate Scholarship from NSERC.

\section{CONFLICT OF INTEREST}

Robert T. Thibault, Michael Lifshitz, and Amir Raz declare that they have no conflict of interest.

\section{INFORMED CONSENT}

All procedures followed were in accordance with the ethical standards of the responsible committee on human experimentation (institutional and national) and with the Helsinki Declaration of 1975, and the applicable revisions at the time of the investigation. Informed consent was obtained from all patients for being included in the study. 


\section{REFERNCES}

Agam, Y., Hämäläinen, M. S., Lee, A. K. C., Dyckman, K. a, Friedman, J. S., Isom, M., et al. (2011). Multimodal neuroimaging dissociates hemodynamic and electrophysiological correlates of error processing. Proceedings of the National Academy of Sciences of the United States of America, 108(42), 17556-61. doi:10.1073/pnas.1103475108

Alperin, N., Hushek, S. G., Lee, S. H., Sivaramakrishnan, A., \& Lichtor, T. (2005). MRI study of cerebral blood flow and CSF flow dynamics in an upright posture: the effect of posture on the intracranial compliance and pressure. Acta neurochirurgica. Supplement, 95, 177-181. http://www.ncbi.nlm.nih.gov/pubmed/16463846

Bastiaansen, M. C. M., \& Knösche, T. R. (2000). Tangential derivative mapping of axial MEG applied to event-related desynchronization research. Clinical Neurophysiology, 111, 13001305. doi:10.1016/S1388-2457(00)00272-8

Berridge, C. W., \& Waterhouse, B. D. (2003). The locus coeruleus-noradrenergic system: modulation of behavioral state and state-dependent cognitive processes. Brain research. Brain research reviews, 42(1), 33-84. http://www.ncbi.nlm.nih.gov/pubmed/12668290

Birn, R. M., Smith, M. a, Jones, T. B., \& Bandettini, P. a. (2008). The respiration response function: the temporal dynamics of fMRI signal fluctuations related to changes in respiration. NeuroImage, 40(2), 644-54. doi:10.1016/j.neuroimage.2007.11.059

Brookes, M., Woolrich, M., Luckhoo, H., Price, D., Hale, Stephenson, M., et al. (2011). Investigating the electrophysiological basis of resting state networks using magnetoencephalography, (16). doi:10.1073/pnas.1112685108

Chang, L.-J., Lin, J.-F., Lin, C.-F., Wu, K.-T., Wang, Y.-M., \& Kuo, C.-D. (2011). Effect of body position on bilateral EEG alterations and their relationship with autonomic nervous modulation in normal subjects. Neuroscience letters, 490(2), 96-100. doi:10.1016/j.neulet.2010.12.034

Cole, R. J. (1989). Postural baroreflex stimuli may affect EEG arousal and sleep in humans. Journal of applied physiology, 67(6), 2369-75. http://www.ncbi.nlm.nih.gov/pubmed/2606843

De Pasquale, F., Della Penna, S., Snyder, A. Z., Lewis, C., Mantini, D., Marzetti, L., et al. (2010). Temporal dynamics of spontaneous MEG activity in brain networks. Proceedings of the National Academy of Sciences of the United States of America, 107(13), 6040-5. doi:10.1073/pnas.0913863107

Deco, G., Jirsa, V. K., \& McIntosh, A. R. (2011). Emerging concepts for the dynamical organization of resting-state activity in the brain. Nature reviews. Neuroscience, 12(1), 4356. doi:10.1038/nrn2961 
Goncharova, I. I., McFarland, D. J., Vaughan, T. M., \& Wolpaw, J. R. (2003). EMG contamination of EEG: Spectral and topographical characteristics. Clinical Neurophysiology, 114(9), 1580-1593. doi:10.1016/S1388-2457(03)00093-2

Gross, J., Baillet, S., Barnes, G. R., Henson, R. N., Hillebrand, A., Jensen, O., et al. (2013). Good practice for conducting and reporting MEG research. NeuroImage, 65, 349-63. doi:10.1016/j.neuroimage.2012.10.001

Hillebrand, A., \& Barnes, G. R. (2002). A quantitative assessment of the sensitivity of wholehead MEG to activity in the adult human cortex. NeuroImage, 16(3 Pt 1),638-650. doi:10.1006/nimg.2002.1102

Jensen, O., Kaiser, J., \& Lachaux, J.-P. (2007). Human gamma-frequency oscillations associated with attention and memory. Trends in neurosciences, 30(7), 317-24. doi:10.1016/j.tins.2007.05.001

Johnson, B. W., Crain, S., Thornton, R., Tesan, G., \& Reid, M. (2010). Measurement of brain function in pre-school children using a custom sized whole-head MEG sensor array. Clinical Neurophysiology, 121(3), 340-349. doi:10.1016/j.clinph.2009.10.017

Jones, D. T., Vemuri, P., Murphy, M. C., Gunter, J. L., Senjem, M. L., Machulda, M. M., et al. (2012). Non-stationarity in the "resting brain's" modular architecture. PloS one, 7(6), e39731. doi:10.1371/journal.pone.0039731

Lei, X., Hu, J., \& Yao, D. (2012). Incorporating FMRI functional networks in EEG source imaging: a Bayesian model comparison approach. Brain topography, 25(1), 27-38. doi:10.1007/s10548-011-0187-9

Lei, X., Xu, P., Luo, C., Zhao, J., Zhou, D., \& Yao, D. (2011). fMRI functional networks for EEG source imaging. Human brain mapping, 32(7), 1141-60. doi:10.1002/hbm.21098

Lipnicki, D. M., \& Byrne, D. G. (2005). Thinking on your back: solving anagrams faster when supine than when standing. Brain research. Cognitive brain research, 24(3), 719-22. doi:10.1016/j.cogbrainres.2005.03.003

Lipnicki, D. M., \& Byrne, D. G. (2008). An effect of posture on anticipatory anxiety. The International journal of neuroscience, 118(2), 227-37. doi:10.1080/00207450701750463

Lundström, J. N., Boyle, J. A., \& Jones-Gotman, M. (2008). Body position-dependent shift in odor percept present only for perithreshold odors. Chemical senses, 33(1), 23-33. doi:10.1093/chemse/bjm059

Mohrman, D. E., \& Heller, L. J. (2003). Cardiovascular Physiology. Lange Medical Books/McGraw-Hill, New York. 
Moradi, F., Liu, L. C., Cheng, K., Waggoner, R. a., Tanaka, K., \& Ioannides, a. a. (2003). Consistent and precise localization of brain activity in human primary visual cortex by MEG and fMRI. NeuroImage, 18(3), 595-609. doi:10.1016/S1053-8119(02)00053-8

Muthukumaraswamy, S. D. (2013). High-frequency brain activity and muscle artifacts in MEG/EEG: a review and recommendations. Frontiers in human neuroscience, 7(April), 138. doi:10.3389/fnhum.2013.00138

Niessing, J., Ebisch, B., Schmidt, K. E., Niessing, M., Singer, W., \& Galuske, R. a W. (2005). Hemodynamic signals correlate tightly with synchronized gamma oscillations. Science (New York, N.Y.), 309(5736), 948-51. doi:10.1126/science.1110948

Nir, Y., Fisch, L., Mukamel, R., Gelbard-Sagiv, H., Arieli, A., Fried, I., \& Malach, R. (2007). Coupling between Neuronal Firing Rate, Gamma LFP, and BOLD fMRI Is Related to Interneuronal Correlations. Current Biology, 17, 1275-1285. doi:10.1016/j.cub.2007.06.066

Okada, Y. C., Wu, J., \& Kyuhou, S. (1997). Genesis of MEG signals in a mammalan CNS sructure. Electroencephalography and clinical neurophysiology, 103, 474-485.

Okamoto, M., Dan, H., Sakamoto, K., Takeo, K., Shimizu, K., Kohno, S., et al. (2004). Threedimensional probabilistic anatomical cranio-cerebral correlation via the international 10-20 system oriented for transcranial functional brain mapping. NeuroImage, 21(1), 99-111. doi:10.1016/j.neuroimage.2003.08.026

Ouchi, Y., Okada, H., Yoshikawa, E., Futatsubashi, M., \& Nobezawa, S. (2001). Absolute Changes in Regional Cerebral Blood Flow in Association with Upright Posture in. Journal of nuclear medicine, 42(5), 707-712.

Ouchi, Y., Yoshikawa, E., Kanno, T., Futatsubashi, M., Sekine, Y., Okada, H., et al. (2005). Orthostatic posture affects brain hemodynamics and metabolism in cerebrovascular disease patients with and without coronary artery disease: a positron emission tomography study. NeuroImage, 24(1), 70-81. doi:10.1016/j.neuroimage.2004.07.044

Poghosyan, V., \& Ioannides, A. a. (2007). Precise mapping of early visual responses in space and time. NeuroImage, 35(2), 759-770. doi:10.1016/j.neuroimage.2006.11.052

Power, J. D., Barnes, K. a., Snyder, A. Z., Schlaggar, B. L., \& Petersen, S. E. (2012). Spurious but systematic correlations in functional connectivity MRI networks arise from subject motion. NeuroImage, 59(3), 2142-2154. doi:10.1016/j.neuroimage.2011.10.018

Ramon, C., Schimpf, P., Haueisen, J., Holmes, M., \& Ishimaru, A. (2004). Role of soft bone, CSF and gray matter in EEG simulations. Brain topography, 16(4), 245-8. http://www.ncbi.nlm.nih.gov/pubmed/15379221 
Rau, H., \& Elbert, T. (2001). Psychophysiology of arterial baroreceptors and the etiology of hypertension. Biological psychology, 57(1-3), 179-201.

http://www.ncbi.nlm.nih.gov/pubmed/11454439

Rehder, K. (1998). Postural changes in respiratory function. Acta anaesthesiologica Scandinavica. Supplementum, 113, 13-6. http://www.ncbi.nlm.nih.gov/pubmed/9932113

Rice, J. K., Rorden, C., Little, J. S., \& Parra, L. C. (2013). Subject position affects EEG magnitudes. NeuroImage, 64, 476-84. doi:10.1016/j.neuroimage.2012.09.041

Shmuel, A., \& Leopold, D. a. (2008). Neuronal correlates of spontaneous fluctuations in fMRI signals in monkey visual cortex: Implications for functional connectivity at rest. Human brain mapping, 29(7), 751-61. doi:10.1002/hbm.20580

Spironelli, C., \& Angrilli, A. (2011). Influence of body position on cortical pain-related somatosensory processing: an ERP study. PloS one, 6(9), e24932.

doi:10.1371/journal.pone.0024932

Stolk, A., Todorovic, A., Schoffelen, J. M., \& Oostenveld, R. (2013). Online and offline tools for head movement compensation in MEG. NeuroImage, 68, 39-48. doi:10.1016/j.neuroimage.2012.11.047

Storey, J. D. (2002). A direct approach to false discovery rates. Journal of the Royal Statistical Society: Series B (Statistical Methodology), 64(3), 479-498. doi:10.1111/1467-9868.00346

Tadel, F., Baillet, S., Mosher, J. C., Pantazis, D., \& Leahy, R. M. (2011). Brainstorm: a userfriendly application for MEG/EEG analysis. Computational intelligence and neuroscience, 2011, 879716. doi:10.1155/2011/879716

Thibault, R. T., Lifshitz, M., Jones, J. M., \& Raz, A. (2014). Posture alters human resting-state. Cortex, 58, 199-205. doi:10.1016/j.cortex.2014.06.014

Vorwerk, J., Cho, J.-H., Rampp, S., Hamer, H., Knösche, T. R., \& Wolters, C. H. (2014). A guideline for head volume conductor modeling in EEG and MEG. NeuroImage, 100, 590607. doi:10.1016/j.neuroimage.2014.06.040

Wendel, K., Narra, N. G., Hannula, M., Kauppinen, P., \& Malmivuo, J. (2008). The influence of CSF on EEG sensitivity distributions of multilayered head models. IEEE transactions on bio-medical engineering, 55(4), 1454-6. doi:10.1109/TBME.2007.912427

Westfall, P., \& Tobias, R. (1999). Advances in multiple comparison and multiple test using the SAS System. In Proceedings of the 24th Annual SAS Users Group International Conference.

Wolters, C. H., Anwander, a., Tricoche, X., Weinstein, D., Koch, M. a., \& MacLeod, R. S. (2006). Influence of tissue conductivity anisotropy on EEG/MEG field and return current 
computation in a realistic head model: A simulation and visualization study using highresolution finite element modeling. NeuroImage, 30, 813-826.

doi:10.1016/j.neuroimage.2005.10.014

Xiang, J., Luo, Q., Kotecha, R., Korman, A., Zhang, F., Luo, H., et al. (2014). Accumulated source imaging of brain activity with both low and high-frequency neuromagnetic signals. Frontiers in neuroinformatics, 8(May), 57. doi:10.3389/fninf.2014.00057

Xu, P., Huang, R., Wang, J., Van Dam, N. T., Xie, T., Dong, Z., et al. (2014). Different topological organization of human brain functional networks with eyes open versus eyes closed. NeuroImage, 90, 246-255. doi:10.1016/j.neuroimage.2013.12.060

Yan, C., Liu, D., He, Y., Zou, Q., Zhu, C., Zuo, X., et al. (2009). Spontaneous brain activity in the default mode network is sensitive to different resting-state conditions with limited cognitive load. PloS one, 4(5), e5743. doi:10.1371/journal.pone.0005743 